\title{
Avances en el desarrollo de nuevas vacunas profilácticas y terapéuticas contra el Virus del Papiloma Humano
}

\author{
Advances in the development of new prophylactic and \\ therapeutic vaccines against Human Papillomavirus
}

\author{
Liliana A. Morales L ${ }^{1}$, Diego F. Rincón R ${ }^{1}$, Bladimiro Rincón Orozco ${ }^{1}$
}

Forma de citar: Morales LA, Rincón DF, Rincón-Orozco B. Avances en el desarrollo de nuevas vacunas profilácticas y terapéuticas contra el Virus del Papiloma Humano. Rev Univ Ind Santander Salud. 2016; 48(3): $385-391$. DOI: http://dx.doi.org/10.18273/revsal.v48n3-2016012 (c) (1) Ð

\section{RESUMEN}

El uso de vacunas profilácticas contra el Virus del Papiloma Humano (VPH) de alto riesgo ha adquirido gran importancia debido al alto potencial oncogénico, de estos virus, especialmente por su asociación con el cáncer de cuello uterino, uno de los canceres más comunes y de mayor mortalidad en mujeres a nivel mundial. El objetivo de este artículo es describir las vacunas profilácticas y terapéuticas disponibles actualmente contra el VPH, analizando las características, ventajas, desventajas y estudios científicos sobre su uso y seguridad en la población. Se llevó a cabo una revisión de literatura de los últimos avances en el desarrollo de vacunas profilácticas y terapéuticas contra el VPH. La vacunación profiláctica es efectiva y esencial para la prevención del cáncer de cuello uterino causadas por VPH-16 y -18, pero no genera protección cruzada contra otros VPH de alto riesgo, esto ha encaminado a la creación de nuevas vacunas nanovalentes que protegen contra un número mas amplio de VPHs de alto riesgo. Por otra parte, las vacunas terapéuticas han demostrado en sus fases de estudio iniciales ser promisorias en la regresión de lesiones premalignas o malignas in situ.

Palabras clave: Vacunas contra HPV, oncogenes, HPV-16, HPV-18, cáncer de cuello uterino.

\begin{abstract}
Prophylactic vaccines against high risk HPV has become important because of the high oncogenic potential of the virus and its association with cervical cancer development, one of the most common and fatal cancers among women worldwide. The objective of this article is describe the state of the art of the current prophylactic and therapeutic HPV vaccines, analyzing their characteristics, advantages, disadvantages and scientific reports on its use and safety in the population. A systematic revision of the latest advances in the development of prophylactic and therapeutic vaccines against HPV was performed. Prophylactic vaccination is effective and essential for the prevention of uterine cervical cancer; new therapeutic vaccines have been shown at the initial phases to be promising contributing in the regression of premalignant or malignant in situ lesions.
\end{abstract}

Keywords: HPV, Human Papilloma Virus, HPV-16, HPV-18, HPV Vaccine, Cervical Cancer.

1. Universidad Industrial de Santander. Bucaramanga, Colombia.

Correspondencia: Bladimiro Rincón Orozco. Dirección: Facultad de Salud, Escuela de Microbiología, Universidad Industrial de Santander. Correo electrónico: blrincon@uis.edu.co. Teléfono: +57 6344000 Ext. 3225. 


\section{INTRODUCCIÓN}

El VPH es un virus desnudo de simetría icosaédrica, cuyo genoma está compuesto por DNA de doble cadena en configuración circular Figura 1A. Su genoma codifica para genes tempranos (Early) entre los que se encuentran E6 y E7 relacionados con la oncogénesis viral y el gen E2 que regula negativamente la expresión de los dos anteriores ${ }^{1-2}$. También posee genes denominados tardíos (Late) donde se encuentran las regiones L1 y L2, siendo L1 el gen que codifica para la proteína estructural principal de la cápside que es la más conservada entre los diferentes tipos de VPH, de ahí que las vacunas profilácticas actuales hayan sido creadas con base en esta proteína $a^{3-4}$.

Los VPH de alto riesgo (VPH-AR) principalmente VPH-16 y -18 tienen un alto potencial oncogénico y se encuentran asociados al cáncer de cérvix, uno de los cánceres más comunes y de mayor mortalidad en mujeres principalmente localizadas en paises en vía de desarrollo. Esta enfermedad presenta en Colombia una tasa de ocho muertes por cada 100.000 habitantes ${ }^{5}$. Estudios recientes han encontrado que la prevalencia de la infección con VPH-AR varía con respecto a la ubicación geográfica así, por ejemplo, la prevalencia del VPH 16/18 combinados en lesiones de cáncer de cuello uterino es levemente mayor en Europa, América del Norte y Australia (74 a 77\%) comparado con África, Asia, América del Sur y América Central (65 a 70\%) $(4,6,7)$. Por su parte otros tipos de VPH-AR como 31 , $33,35,39,45,52$ y 58 tienen una menor prevalencia a nivel mundial y se encuentran asociados a un $20 \%$ a los casos de cáncer de cérvix ${ }^{6-7}$.

\section{METODOLOGÍA}

Este estudio tuvo un enfoque de tipo cualitativo, interpretativo-descriptivo y un diseño no experimental. Se llevó a cabo una revisión de literatura con documentos bibliográficos especializados publicados en revistas indexadas.

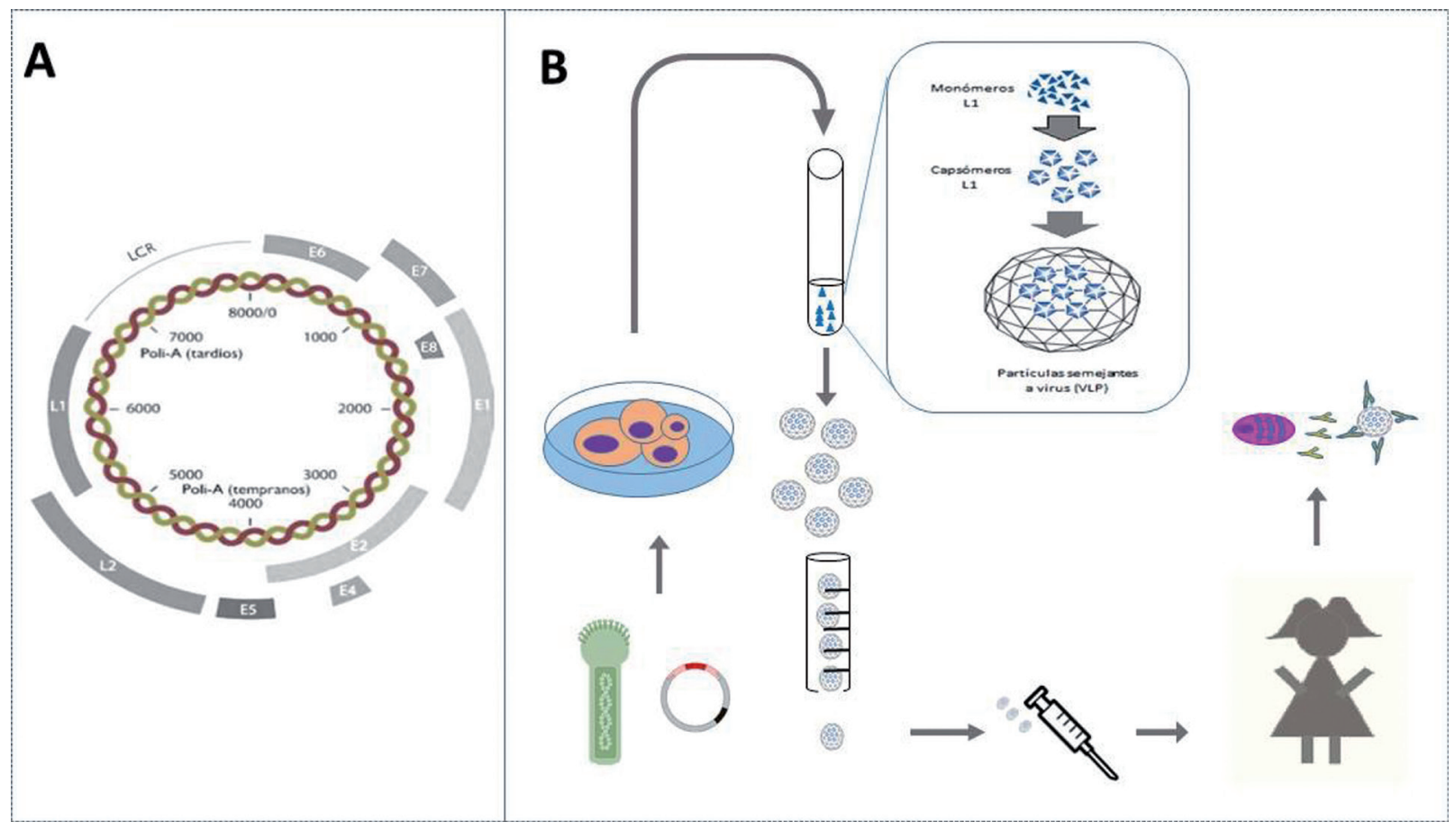

Figura 1. A. Organización del genoma del VPH, adaptada de Soberón, A 2006; ${ }^{10}$ B. VLPs; adaptada de: Schiller, J \& Müller, M 2015. ${ }^{11}$ Proceso de síntesis de las vacunas profilácticas contra el VPH adaptado de Bersosky JA, et al 2004. ${ }^{12}$ Durante la síntesis se utilizan proteínas recombinantes de la proteína mayor de la cápside (L1) del VPH, morfológicamente idénticas a los viriones originales, mediante la introducción del gen de la misma en cultivos de células eucariotas y, posteriormente, su capacidad de autoensamblaje formando unas estructuras tridimensionales vacías llamadas VLPs que son purificadas y que posteriormente pueden ser utilizadas para inmunizar a los individuos que después de la vacunación generarán anticuerpos neutralizantes protectores $^{12}$. 


\section{Lo que se debe saber sobre la vacunación profiláctica contra el VPH}

Las vacunas contra el VPH han sido desarrolladas con base en el sistema de partículas semejantes a virus (Virus Like Particles: VLPS) Figura 1B, que consiste en clonar la L1 (proteína mayoritaria de la cápside), y colocarla en condiciones óptimas que permiten autoensamblaje formando así las VLPs morfológicamente idénticas al virus, pero carentes del genoma viral Figura $1 \mathbf{B}^{11}$. Las VLPs de L1 han demostrado tener una potente capacidad antigénica y alta seguridad, incluso, se ha observado en diferentes estudios que la estabilidad de la respuesta inmunogénico perdura por más de 10 años ${ }^{13-14}$.

Hasta la fecha la Agencia de Alimentos y Medicamentos de Estados Unidos (U.S. Food and Drug Administration: FDA) ha aprobado el uso de tres vacunas profilácticas contra el VPH, la vacuna bivalente $\left(\right.$ Cervarix $^{\circledR}$ ) producida por GlaxoSmithKline Biologicals $\mathrm{SA}^{\circledR}$ y la vacuna tetravalente $\left(\right.$ Gardasi $\left.^{\circledR}\right)$ y nonavalente $\left(\right.$ Gardasil $\left.{ }^{\circledR}\right)$ producidas por Merck ${ }^{\circledR}$ las cuales tienen adyuvantes que permiten la lenta presentación del antígeno viral por parte de los monocitos hacia los linfocitos B (Tabla 1) ${ }^{15}$. Estas vacunas se han asociado con una reducción en la incidencia de la infección, verrugas genitales y cáncer cervical ${ }^{11,16}$.

La vacuna bivalente se encuentra dirigida contra los tipos de VPH de alto riesgo 16 y 18; es construida en una línea celular de insecto infectada con un vector de baculovirus llamado Trichoplusia ni y contiene como adyuvantes el ASO4 (hidróxido de aluminio) y el lípido A monofosforilado (MPL, monophosphoryl lipid A), tiene la facultad de activar la respuesta inmune inespecífica mediante el receptor 4 tipo Toll $<$ TLR4, Tolllike receptor $4>.{ }^{11,15} \mathrm{La}$ vacuna bivalente ha demostrado gran eficacia contra las infecciones persistentes y lesiones de alto grado. Estudios han estimado que independientemente del tipo de VPH la vacuna bivalente presenta un $93,2 \%$ de eficacia contra lesiones intraepiteliales que sean igual o mayor a grado tres (Cervical Intra-epithelial Neoplasia 3 CIN 3) ${ }^{17}$.

En cuanto a la vacuna tetravalente esta es recomendada por la FDA desde el 2006 para su uso tanto en hombres como mujeres y para que sea eficaz este biológico debe ser, como todas las vacunas de su tipo, aplicado antes de la primera relación sexual ${ }^{18}$; se encuentra dirigida contra dos genotipos de VPH-AR (16 y 18) y dos VPH de bajo riesgo (VPH-BR) (6 y 11). Esta vacuna es producida en un sistema de levadura denominado Saccharomyces cerevisiae y tiene como adyuvante AAHS (sulfato de hidroxifosfato aluminio amorfo $)^{2}$. Los dos estudios de fase III para la vacuna tetravalente denominados FUTURE-I y FUTURE-II encontraron que la eficacia de esta vacuna fue estadísticamente significativa al 100\% para CIN 2 y AIS (adenocarcinoma in situ) relacionado con los tipos de HPV-16, -18 y un 96,8\% para CIN $3^{13}$.

Recientemente la FDA aprobó en hombres y mujeres el uso de Gardasi19 ${ }^{\circledR}$, vacuna nonavalente dirigida contra los tipos de VPH-BR $(6,11)$ y VPH-AR $(16,18,31$, $33,45,52$ y 58$)$, siendo estos últimos los responsables de aproximadamente el 90\% de los canceres de cérvix, vulva, vagina y ano. Esta vacuna es una nueva versión de la vacuna tetravalente producida por Merck y se ha desarrollado bajo sus mismos principios representando un avance importante en la prevención del cáncer ya que protege adicionalmente contra los tipos de VPH $31,33,45,52$ y 58 , que causan alrededor del $20 \%$ de los canceres cervicales y que no se encuentran cubiertos por las dos vacunas descritas anteriormente, adicionalmente esta vacuna también previene las verrugas genitales causadas por los tipos de VPH-6 y -11 16,19-21.

A nivel mundial se han realizado estudios de seguridad en fase III, en los cuales más de 20.000 mujeres entre los 9-26 años recibieron la vacuna tetravalente y más de 30.000 mujeres a partir de los 10 años fueron inmunizadas con la vacuna bivalente. Posteriormente se hicieron estudios de seguridad después de su aprobación en donde a finales de 2013, más de 144 millones de dosis de la vacuna tetravalente y cerca de 41 millones de dosis de la bivalente han sido distribuidas a nivel mundial. Los efectos secundarios más comunes observados durante dichos estudios incluyeron dolor, rubor y sincope. En cuanto a la seguridad, no se han observado diferencias significativas en la aparición de enfermedades autoinmunes y enfermedades crónicas o muertes relacionadas posterior a vacunación contra $\mathrm{VPH}^{10}$.

Un aspecto importante con respecto a la respuesta inmune entre las dos vacunas es que Gardasil ${ }^{\circledR}$ induce únicamente una respuesta $\mathrm{TH} 2$ que genera la producción de anticuerpos neutralizantes y Cervarix $^{\circledR}$ induce anticuerpos neutralizantes combinado con activación de linfocitos ayudadores T1 (TH1), que produce una respuesta potente de células T CD8, sin embargo debido a las características del ciclo viral de VPH no se obtiene una respuesta protectora por parte de los linfocitos $\mathrm{T}$, lo que hace que la protección de estas vacunas recaiga completamente sobre la producción de anticuerpos neutralizantes contra el virus ${ }^{15}$.

Recientemente, el uso de vacunas profilácticas contra 
los VPH-AR-16 y -18 ha adquirido mayor importancia, pero es importante mencionar acerca de los hechos ocurridos en el Carmen de Bolívar (Bolívar, Colombia) que generaron gran incertidumbre y dudas en los colombianos con respecto a la aplicación y seguridad sobre la vacunacion contra VPH, dado que más de 500 niñas que fueron vacunadas presentaron una variedad de síntomas que han sido descritos por varios autores como "Evento Psicogénico Masivo", ya que no hay evidencia suficiente sobre su relación con la vacuna pero que al ser altamente difundido en los medios de comunicación ha generado efectos nocivos para el programa nacional de vacunación ${ }^{8-9}$. Lo anterior es apoyado por los estudios realizados alrededor del mundo donde se evaluó la seguridad de las diferentes vacunas profilácticas disponibles en el mercado con resultados muy positivos.

De hecho, para confirmar la seguridad de la vacuna en Colombia, en el 2004 aproximadamente 200 adolescentes recibieron tres dosis de la vacuna tetravalente en un protocolo de investigación de casos y controles en Cali, Medellín y Bogotá, realizando desde entonces un seguimiento muy estricto cada seis meses en los cuales solo se han presentado eventos menores (dolor, rubor en el sitio de la inyección, fiebre, sincope) semejantes a los que se presentan con las demás vacunas ${ }^{9}$.

\section{Avances en la vacunación terapéutica contra VPH como adyuvante a procedimientos invasivos}

La infección persistente VPHs-AR en la población susceptible se ve favorecida debido a un desarrollo ineficaz de la respuesta inmune innata y celular adaptiva que sería la responsable de eliminar o controlar la infección viral crónica en el interior de los queratinocitos ${ }^{16,22}$. Por otra parte, a pesar de la efectividad de las vacunas profilácticas estas no tienen actividad en infecciones establecidas. Esta es la principal razón por la cual en los últimos años se han venido desarrollando vacunas terapéuticas contra el VPH utilizando las oncoproteínas E6 y E7 como los principales blancos, ya que son las más oncogénicas y son esenciales para el mantenimiento del tumor Tabla $\mathbf{1}^{2,17}$.

Tabla 1. Características de las actuales vacunas profilácticas y terapéuticas contra el VPH.

\begin{tabular}{|c|c|c|c|c|c|c|c|c|}
\hline \multirow[b]{2}{*}{$\begin{array}{l}\text { Vacunas } \\
\text { Profilácticas }\end{array}$} & \multirow[b]{2}{*}{ Antígeno } & \multirow[b]{2}{*}{ Adyuvante } & \multicolumn{2}{|l|}{ Tipo de VPH } & \multirow{2}{*}{$\begin{array}{c}\text { Vía de } \\
\text { aplicación }\end{array}$} & \multirow[b]{2}{*}{ Dosis } & \multirow[b]{2}{*}{ Fase } & \multirow[b]{2}{*}{ Referencia } \\
\hline & & & Alto riesgo & $\begin{array}{c}\text { Bajo } \\
\text { riesgo }\end{array}$ & & & & \\
\hline Cervarix $^{\circledR}$ & L1 & AS04 & 16 y 18 & & IM & 3 & III finalizada & (11) (15) \\
\hline Gardasil $^{\circledR}$ & L1 & AAHS & 16 y 18 & $6 \mathrm{y} 11$ & IM & 3 & III finalizada & $(2)(15)$ \\
\hline Gardasil9 $^{\circledR}$ & L1 & AAHS & $16,18,31,33,45,52$ у 58 & 6 y 11 & IM & 3 & III finalizada & $(11)(15)(19)$ \\
\hline \multicolumn{9}{|l|}{$\begin{array}{l}\text { Vacunas } \\
\text { terapéuticas }\end{array}$} \\
\hline VGX3100 & E6/E7 & Ninguno & 16,18 & & IM & 3 & II & $(30)$ \\
\hline TG4001 & E6/E7 & L2 & 16 & & $\mathrm{SC}$ & 3 & II & $(23)(27)$ \\
\hline GX-188 & E6/E7 & Flt3L & 16 y 18 & & IM & 3 & I & $(31)$ \\
\hline E7-mHsp70 & E7 & Ninguno & 16 & & IV-IP-IV* & $3^{*}$ & Preclínica & $(27)(28)$ \\
\hline Pentarix & E7 & TLR3/TLR9 & $16,18,31,45$ y 52 & & $\mathrm{SC}^{*}$ & $1-4^{*}$ & Preclínica & $(26)(27)$ \\
\hline
\end{tabular}

ASO4: lípido A monofosforilado e hidróxido de aluminio; AAHS: sulfato de hidroxifosfato de aluminio amorfo; Fit3L: Fms-like tyrosine kinase-3 ligand; TLR: receptores tipo Toll; L1: proteína mayor de la cápside; L2: proteína menor de la cápside; E5/E7: oncoproteínas virales; IM: intramuscular; SC: subcutánea; IV: intravenosos; IP: intraperitoneal;

*Ensayos realizados en modelo murino.

Entre las tecnologías usadas para la creación de este tipo de vacunas se encuentran aquellas basadas en vectores vivos atenuados, que incluyen virus o bacterias. Estos tienen como ventaja que son capaces de replicarse y por lo tanto propagar los antígenos, además pueden inducir la presentación de antígenos llevando las oncoproteínas del VPH a las células dendríticas. Sin embargo, la generación de anticuerpos neutralizantes constituye la mayor desventaja e incluso la posibilidad que exista una inmunidad previa contra el vector utilizado; también existe el riesgo inherente que implica el uso de estos 388 microorganismos atenuados como vectores sobre todo en pacientes inmunocomprometidos ${ }^{3,23,-24}$.

Un ejemplo de vacuna basada en vectores vivos es la TG4001, una vacuna terapéutica que usa como vector el virus de la vaccinia altamente atenuado, es diseñada para expresar antígenos E6 y E7 del VPH-16 y usa la proteína estructural L2 del VPH como coadyuvante. En estudios de fase II esta vacuna se asoció con una baja morbilidad e indujo la regresión de CIN 2 y 3 relacionados con el VPH- $16^{25}$. 
También existen ensayos con vacunas que han mostrado ser seguras y fáciles de producir basadas en péptidos, proteínas y unos híbridos de células dendríticas y células tumorales. Estudios preclínicos han indicado que los péptidos E6 y E7 son capaces de inducir una respuesta de células $\mathrm{T}$ específica sin embargo, debido a su pobre inmunogenicidad deben ser usados junto con coadyuvantes. En 2011 Jing Zhang, et al. desarrollaron la vacuna Pentarix (con TLR3/TLR9 como coadyuvantes) dirigida contra las proteínas E7 de cinco genotipos de VPH-AR de mayor prevalencia en todo el mundo (16, $18,31,45$ y 52 ), los cuales representan más del $80 \%$ de todos los cánceres asociados al VPH; esta vacuna ha demostrado eficacia en modelos preclínicos murinos de cáncer inducido por $\mathrm{VPH}^{26-27}$. En un estudio diferente se demostró que la inmunización en ratones (fase preclínica) con la proteína E7-mHsp70 (proteína murina de choque térmico de $70 \mathrm{kDa}$ ) sin ningún adyuvante genera respuesta inmune anti-tumoral en ratones ${ }^{28}$.

Un aspecto novedoso en la inmunidad antitumoral es la estimulación de células dendríticas (DC: dendritic cells) con un fenotipo más inmunogénico contra los tumores VPH positivos, estas DC pueden estimular de manera más eficiente la respuesta de los linfocitos $\mathrm{T}$ citotóxicos $(\mathrm{LT} \mathrm{CD} 8+)^{29}$. Esto se debe a que las DC potencian la respuesta inmune mediante diferentes señales como moléculas co-estimuladoras, presentación antigénica del complejo péptido-MHC y citocinas necesarias para que la activación de células $\mathrm{T}$ vírgenes (naïve) desarrollen respuestas celulares adaptativas más eficientes ${ }^{30}$. Varios estudios clínicos han mostrado que la incubación y activación inicial (priming) de DC y la proteína E6/E7 del HPV-18 indujeron respuestas de células específicas de antígeno CD4 + y CD8, mostrando una mejoría clínica en pacientes con cáncer cervical ${ }^{31}$.

Otras propuestas incluyen modelos terapéuticos basados en vectores de ADN que emplean la inyección directa de plásmidos, estos codifican para los oncogenes E6 y E7 de los VPH-16 y -18 en el hospedero, induciendo la expresión y presentación de los antígenos codificados por las células transfectadas promoviendo así la respuesta inmune celular. Estos vectores son fáciles de fabricar y son más seguros en comparación con los vectores vivos, además no aumentan la resistencia a la vacuna porque no estimulan la producción de anticuerpos siendo la pobre inmunogenicidad su principal desventaja ${ }^{32}$.

Recientemente Trimble et al. evaluaron el uso de la vacuna VGX3100 en fase clínica II. Esta vacuna está basada en plásmidos con las oncoproteínas E6 y E7 tanto del VPH-16 y -18, y se aplica utilizando un dispositivo llamado CELLECTRAel cual genera impulsos eléctricos controlados en el sitio de inyección, que aumentan la permeabilidad de la membrana celular y la transferencia intracelular de VGX-3100 al núcleo, lo que resulta en una mayor transfección, generación de las proteínas oncogenicas y la subsiguiente inmunogenicidad. Esta vacuna podría presentar una opción terapéutica no quirúrgica cambiando las perspectivas del tratamiento común ya que es la primera vacuna en mostrar eficacia contra lesiones CIN 2 y 3 asociadas a los HPV-16 y $-18^{33}$. También se ha documentado la vacuna GX-188, que consiste en una vacuna de DNA diseñada para facilitar el procesamiento y presentación de antígenos E6 y E7 de los VPH-16 y -18 por células dendríticas y en los estudios realizados fue capaz de inducir una fuerte respuesta inmune mediada por células $\mathrm{T}$, demostrando ser eficaz y capaz de erradicar las lesiones asociadas a $\mathrm{VPH}^{34}$.

\section{CONCLUSIONES}

Claramente el programa de vacunación en Colombia enfrenta un mal momento debido a la desinformación por parte de los medios, aunque existan evidencias científicas sólidas generadas por organismos serios e imparciales a nivel internacional que sustentan la seguridad de la vacunación y la importancia de su aplicación en edades tempranas.

Por otra parte, si bien la vacunación profiláctica es efectiva y en la actualidad su uso sigue siendo esencial para la prevención del cáncer de cérvix y otros tipos de cáncer asociados al VPH, tanto en hombres como en mujeres. Esta vacuna tiene ciertas desventajas en cuanto a costos, la no generación de protección cruzada contra todos los VPH-AR y la falta de efectividad en individuos previamente infectados con VPH, lo que ha encaminado a la creación de nuevas vacunas terapéuticas que aunque se encuentran en ensayos preclínicos y fases clínicas tempranas han demostrado ser promisorias en la regresión de lesiones asociadas a esta infección viral, convirtiendose además en una promisoria terapia complementaria a los tratamientos oncologicos actuales.

\section{REFERENCIAS}

1. Doorbar J. The papillomavirus life cycle. J Clin Virol. 2005; 32(Suppl 1): 7-15.

2. Haupt RM, Sings HL. The Efficacy and Safety of the Quadrivalent Human Papillomavirus 6/11/16/18 Vaccine Gardasil. J Adolesc Heal. 2011; 49(5): 467475. DOI: $10.1016 /$ j.jadohealth.2011.07.003 
3. Kumar S, Biswas M, Jose T. HPV vaccine: Current status and future directions. Med journal, Armed Forces India. 2015;71(2):171-177. DOI: 10.1016/j. mjafi.2015.02.006.

4. Morshed K, Polz-Gruszka D, Szymański M, PolzDacewicz M. Human Papillomavirus (HPV) - Structure, epidemiology and pathogenesis. Otolaryngol Pol. 2014; 68(5): 213-219. DOI:10.1016/j.otpol.2014.06.001

5. Dimaio D. Nuns, Warts, Viruses, and Cancer. J Biol Med. 2015; 88:127-9.

6. Smith JS, Lindsay L, Hoots B, Keys J, Franceschi $\mathrm{S}$, Winer $\mathrm{R}$, et al. Human papillomavirus type distribution in invasive cervical cancer and highgrade cervical lesions: a meta-analysis update. Int J Cancer. 2007; 121(3): 621-632.

7. Wang HY, Park S, Lee D, Kim S, Kim G, Park KH, et al. Prevalence of type-specific oncogenic human papillomavirus infection assessed by HPV E6/E7 mRNA among women with high-grade cervical lesions. Int J Infect Dis. 2015; 37:135-142. DOI: 10.1016/j.ijid.2015.06.018.

8. Grillo-Ardila CF. El Carmen de Bolivar: Una lección que no debemos olvidar. Rev Colomb Obstet Ginecol. 2014; 65(3): 198-201.

9. Reina J c, Muñoz N. Vacuna contra el virus del papiloma humano. Colomb Med. 2014; 45(3): 9495.

10. Lopez-Saavedra A, Lizano-Soberón M. Cáncer cérvicouterino y el virus del papiloma humano: La historia que no termina. Cancerología. 2006; 1: 3155.

11. Schiller JT, Müller M. Next generation prophylactic human papillomavirus vaccines. Lancet Oncol. 2015; 16(5): e217-225. DOI: http://dx.doi. org/10.1016/S1470-2045(14)71179-9.

12. Berzofsky JA, Ahlers JD, Janik J, Morris J, Oh S, Terabe $\mathrm{M}$, et al. Progress on new vaccine strategies against chronic viral infections. J Clin Invest. 2004; 114(4): 450-462.

13. Lowy DR, Herrero R, Hildesheim A. Primary endpoints for future prophylactic human papillomavirus vaccine trials: towards infection and immunobridging. Lancet Oncol. 2015; 16(5): e226233. DOI: 10.1016/S1470-2045(15)70075-6.

14. Pils S, Joura EA. From the monovalent to the ninevalent HPV vaccine. Clin Microbiol Infect. 2015; 21(9): 827-833. doi: 10.1016/j.cmi.2015.05.001.

15. Schiller JT, Lowy DR. Understanding and learning from the success of prophylactic human papillomavirus vaccines. Nat Rev Microbiol. 2012;10(10): 681-692. DOI: 10.1038/nrmicro2872.

16. Herrero R, González P, Markowitz LE. Present status of human papillomavirus vaccine development and implementation. Lancet Oncol. 2015;16(5):e206216. DOI: http://dx.doi.org/10.1016/S14702045(14)70481-4.

17. Giannini SL, Boxus M, Lockman L, Fochesato M, Lorin C, Thomas F. Antibody avidity measurements in recipients of Cervarix ${ }^{\circledR}$ vaccine following a two-dose schedule or a three-dose schedule. Vaccine. 2014; 32(26): 3232-3236. DOI: 10.1016/j. vaccine.2014.04.005.

18. U.S. Food and Drug Administration. Gardasil. 2015

19. Kirby T. FDA approves new upgraded Gardasil 9. Lancet Oncol. 2014;16(2):e56. DOI: 10.1016/ S1470-2045(14)71191-X.

20. U.S. Food and Drug Administration. FDA approves Gardasil 9 for prevention of certain cancers caused by five additional types of HPV.

21. Dochez C, Bogers JJ, Verhelst R, Rees H. HPV vaccines to prevent cervical cancer and genital warts: an update. Vaccine. 2014; 32(14): 1595-601. DOI: $10.1016 /$ j.vaccine.2013.10.081.

22. Stanley M. HPV - immune response to infection and vaccination. Infect Agent Cancer. 2010; 5(1): 19. DOI: 10.1186/1750-9378-5-19

23. Bolhassani A, Mohit E, Rafati SR. Different spectra of therapeutic vaccine development against HPV infections. Hum Vaccin. 2009; 5(10): 671-689.

24. Adachi K, Kawana K, Yokoyama T, Fujii T, Tomio A, Miura S, et al. Oral immunization with a Lactobacillus casei vaccine expressing human papillomavirus (HPV) type $16 \mathrm{E} 7$ is an effective strategy to induce mucosal cytotoxic lymphocytes against HPV16 E7. Vaccine. 2010; 28(16): 28102817. DOI: 10.1016/j.vaccine.2010.02.005.

25. Brun J-L, Dalstein V, Leveque J, Mathevet P, Raulic P, Baldauf J-J, et al. Regression of high-grade cervical intraepithelial neoplasia with TG4001 targeted immunotherapy. Am J Obstet Gynecol. 2011;204(2):169.e1-8.

26. Webb JR, Wick DA. A novel, broad spectrum therapeutic HPV vaccine targeting the E7 proteins of HPV16, 18, 31, 45 and 52 that elicits potent E7-specific CD8T cell immunity and regression of large, established, E7-expressing TC-1 tumors. Vaccine. 2011; 29(44): 7857-7866. DOI: 10.1016/j. vaccine.2011.07.090.

27. Lin J, Xu J, Albers AE, Kaufmann AM. New Developments in Therapeutic HPV Vaccines. Curr Obstet Gynecol Rep. 2012; 1(3): 106-115. DOI: 10.1007/s13669-012-0015-6.

28. Li Y-L, Liu J, Liu J-N, Zhang J. Immunization of protein HPV16 E7 in fusion with mouse HSP70 inhibits the growth of TC-1 cells in tumor bearing 
mice. Vaccine. 2011; 29(35): 5959-5962. DOI: 10.1016/j.vaccine.2011.06.046.

29. Li J, Li J, Aipire A, Luo J, Yuan P, Zhang F. The combination of Pleurotus ferulae water extract and CpG-ODN enhances the immune responses and antitumor efficacy of HPV peptides pulsed dendritic cell-based vaccine. Vaccine. 2016; S0264-410X(16): 30312-30317. DOI: 10.1016/j.vaccine.2016.05.022.

30. Garcia-Bates TM, Kim E, Concha-Benavente F, Trivedi S, Mailliard RB, Gambotto A, et al. Enhanced Cytotoxic CD8 T Cell Priming Using Dendritic Cell-Expressing Human Papillomavirus-16 E6/ E7-p16INK4 Fusion Protein with Sequenced AntiProgrammed Death-1. J Immunol. 2016; 196(6): 2870-2878. DOI: 10.4049/jimmunol.1502027.

31. Yang MC, Yang A, Qiu J, Yang B, He L, Tsai YC, et al. Buccal injection of synthetic HPV long peptide vaccine induces local and systemic antigen specific $\mathrm{CD} 8+\mathrm{T}$ cell immune responses and antitumor effects without adjuvant. Cell Biosci. 2016; 6(17): 1-10. DOI: $10.1186 / \mathrm{s} 13578-016-0083-9$

32. Lin K, Doolan K, Hung C-F, Wu TC. Perspectives for Preventive and Therapeutic HPV Vaccines. J Formos Med Assoc. 2010; 109(1): 4-24.

33. Trimble CL, Morrow MP, Kraynyak KA, Shen X, Dallas M, Yan J, et al. Safety, efficacy, and immunogenicity of VGX-3100, a therapeutic synthetic DNA vaccine targeting human papillomavirus 16 and 18 E6 and E7 proteins for cervical intraepithelial neoplasia 2/3: a randomised, double-blind, placebo-controlled phase $2 \mathrm{~b}$ trial. 2015; 386(10008): 2078-20881-11. DOI: http:// dx.doi.org/10.1016/S0140-6736(15)00239-1

34. Kim TJ, Jin H-T, Hur S-Y, Yang HG, Seo YB, Hong $\mathrm{SR}$, et al. Clearance of persistent HPV infection and cervical lesion by therapeutic DNA vaccine in CIN3 patients. Nat Commun. 2014; 5:5317. DOI: 10.1038/ncomms6317. 